\title{
The neurotoxicity of DE-71: effects on neural development and impairment of serotonergic signaling in zebrafish larvae
}

\author{
Xianfeng Wang ${ }^{\mathrm{a}, \mathrm{b}}$, Lihua Yang ${ }^{\mathrm{a} *}$, Qiangwei Wangc, Yongyong Guo ${ }^{\mathrm{a}}, \mathrm{Na} \mathrm{Li}^{\mathrm{d}}$, \\ Mei Mad and Bingsheng Zhou ${ }^{\mathrm{a}}$
}

\begin{abstract}
The underlying mechanism of polybrominated diphenyl ether (PBDE)-induced neurotoxicity is still a major concern due to its ubiquitous nature and persistence. Here, zebrafish embryos ( $2 \mathrm{~h}$ postfertilization, hpf) were exposed to different concentrations of the commercial PBDE mixture DE-71 $\left(0-100 \mu \mathrm{gl}^{-1}\right)$ until $120 \mathrm{hpf}$, and the impact on neural development and serotonergic system was investigated. The in vivo results revealed significantly reduced transcription of genes involved in neurogenesis (fgf8, shha, wnt1), and contents of proteins in neuronal morphogenesis (myelin basic protein, synapsin Ila), suggesting an impairment of neural development in zebrafish embryos. Further results demonstrated a reduction of 5-hydroxytryptamine neuron and a dose-dependent decrease of whole-body serotonin levels, as well as the transcription of genes involved in serotonergic synthesis (tph1, tph2, trhr) and neurotransmission (serta/b, htr1aa/b). In addition, we predicted possible targets of PBDEs by molecular docking, and the results indicated that PBDE congeners showed high binding affinities with fibroblast growth factor 8 other than SHH and HTR1B. Taken together, this study demonstrated that PBDE exposure during embryogenesis could damage neural development and cause impairment of the serotonergic system as secondary effects in the zebrafish larvae. Copyright @ 2016 John Wiley \& Sons, Ltd.
\end{abstract}

Keywords: PBDEs; zebrafish larvae; neural development; serotonergic system; molecular docking

\section{Introduction}

Polybrominated diphenyl ethers (PBDEs) are among the most persistent organic pollutants, and have caused great concern during the past decades, as they have been shown to disturb thyroid hormone homeostasis due to structural similarity (Dingemans et al., 2011). PBDEs have also been repeatedly reported to affect the central nervous system and cause permanent impairment in spontaneous behavior (locomotion, rearing and total activity), habituation capability as well as performance in learning tasks in rodents (Costa \& Giordano, 2007; Dingemans et al., 2011; Eriksson et al., 2001). Some previous studies have attributed PBDE-induced neurotoxicity to indirect effects following thyroid disruption, while others have proposed direct effects of neuronal signaling, cell migration, axon formation, synaptic plasticity, ion channels, calcium homeostasis and neurotransmission (Dingemans et al., 2011; Kodavanti \& Ward, 2005; Mariussen \& Fonnum, 2003). The mechanism of toxicity of PBDEs, however, still needs to be clarified.

To the best of our knowledge, of the key events during early neural development, three inductive signaling factors, including fibroblast growth factors (FGF8), Sonic hedgehog (SHH) and Wingless/int-1 (WNT1), have been demonstrated to collaborate in inducing the neural plate (Hynes et al., 2000; Ye et al., 1998), and the proliferation and differentiation of neuronal progenitors (Hirabayashi et al., 2004; Muroyama et al., 2004; Viti et al., 2003). While in neuronal morphogenesis, the role of myelin basic protein (MBP) and synapsin Ila (SYN2a) were well studied. MBP is considered a biomarker of myelination that is required for the myelination of axons in the developing central nervous system in zebrafish (Brosamle \& Halpern, 2002) under the regulation of $\mathrm{SHH}$ (Chen et al., 2013a). SYN2a is a biomarker of synapse formation, which plays an important role in both synaptogenesis and neurotransmitter release (Kao et al., 1998); therefore, we presume that PBDEs could affect early development through these signalings to induce neurotoxicity. Although not specific, the FGF, SHH and WNT1 signalings have been proven to play critical roles in the specification and differentiation of monoamine neurons (Hynes et al., 2000; Ye et al., 1998). Hence, it is very likely that the monoamine serotonin (5-hydroxytryptamine, 5-HT) could also be affected. Serotonin is synthesized through the actions of different tryptophan hydroxylases, $\mathrm{TpH} 1, \mathrm{TpH} 2$ and $\mathrm{TpHR}$. The serotonin transporter (SERT) and serotonin receptors play important roles in synaptic 5-HT neurotransmission: SERT acts as both sym- and antiporter in the presynaptic membrane, responsible for removal and reuptake of serotonin from the synapse (Gershon \& Tack, 2007; Wang et al., 2006). Knockout of 5-HT transporter in rats could alter the brain serotonin homeostasis (Bengel et al., 1998; Olivier et al., 2008). The G-protein-coupled receptor, HTR1A, may act as an

\footnotetext{
* Correspondence to: L. H. Yang, Institute of Hydrobiology, Chinese Academy of Sciences, Wuhan 430072, China.

E-mail: Ihyang@ihb.ac.cn

${ }^{a}$ State Key Laboratory of Freshwater Ecology and Biotechnology, Institute of Hydrobiology, Chinese Academy of Sciences, Wuhan 430072, China

${ }^{b}$ University of Chinese Academy of Sciences, Beijing 100039, China

'Institute of Pesticide and Environmental Toxicology, Zhejiang University, Hangzhou 310058, China

${ }^{d}$ State Key Laboratory of Environmental Aquatic Chemistry, Research Center for Eco-Environmental Sciences, Chinese Academy of Sciences, Beijing 100085, China
} 
autoreceptor in a presynaptic negative-feedback loop of 5-HT, and propagate serotoninergic signaling postsynaptically (Masson et al., 2012; Norton et al., 2008). In addition to its critical role in the modulation of behaviors (Airhart et al., 2012; Schaefer et al., 2009), $5-\mathrm{HT}$ is of particular interest because it contributes to promote the specification, differentiation and phenotype maintenance of the hippocampal neurons via activation of its receptors (Fricker et al., 2005). Therefore, it must be interesting whether PBDEs could directly interfere with genes or proteins involved in serotonergic signaling and cause corresponding effects to neural development.

Zebrafish have been shown to be a useful model for studying the mechanism of neurotoxicity by means of neural developmental, neurochemical and neurobehavioral changes (Eddins et al., 2010). The definition of the molecular target as initial events for the in vivo observations could be another challenge. For this intension, in silico approaches such as molecular docking are suitable tools to fill gaps in knowledge concerning chemical and biological levels. The docking methodologies were based on the knowledge of three-dimensional structure of a receptor protein in attempt to optimize the bound ligand or a series of molecules into the active site. This approach can facilitate identification of the initial step for chemical molecular targets by estimation of receptor-ligand binding modes and binding affinities, and has been widely accepted as a potent tool in toxicological studies (Li et al., 2010, 2013). Therefore, the main purpose of the present study was to evaluate the effects on neural developmental and serotonergic system in zebrafish embryo/larvae upon exposure to the PBDE mixture, DE-71, and predicted the possible targets by molecular docking.

\section{Materials and methods}

\section{Chemicals}

DE-71 (purity $>99.9 \%$ ) was obtained from Wellington Laboratory (Ontario, Canada). 5-HT was purchased from Sigma-Aldrich (St. Louis, MO, USA). The PBDEs standards (BDE-28, BDE-47, BDE-71, BDE-99, BDE-153, BDE-154), ${ }^{13} \mathrm{C}_{12}$-labeled BDE-139 and ${ }^{13} \mathrm{C}_{12}$-labeled PCB-208 were purchased from AccuStandard (St. Louis, MO, USA). Trizol reagent and PrimeScript reverse transcription (RT) reagent kits were purchased from Takara (Dalian, China). SYBR Green polymerase chain reaction (PCR) kits were purchased from GeneCopoeia (Rockville, MD, USA). All other chemicals used in the present study were analytical grade standard.

\section{Zebrafish embryos/larvae exposure}

Adult wild-type zebrafish ( $A B$ strain, 5 months old) maintenance and embryos exposure were carried out according to published protocols (Yu et al., 2010). Briefly, 2000 normal developing embryos that reached the blastula stage ( $2 \mathrm{~h}$ post-fertilization, $\mathrm{hpf}$ ) were selected and distributed into glass beakers containing $500 \mathrm{ml} \mathrm{DE-71} \mathrm{exposure} \mathrm{solutions}\left(0,3,10,30\right.$ or $\left.100 \mu \mathrm{gl}^{-1}\right)$ until $120 \mathrm{hpf}$. Both the control and exposure groups received $0.005 \%$ $(\mathrm{v} / \mathrm{v})$ dimethyl sulfoxide, and the exposure solutions were renewed daily. After exposure, a subset of the larvae were used for locomotor activity measurement, whereas the others were washed five times, immediately frozen in liquid nitrogen and stored at $-80^{\circ} \mathrm{C}$ until for analysis. The hatching, malformation and growth were also recorded.

\section{Polybrominated diphenyl ether extraction and analysis in zebrafish larvae}

For PBDE analysis, $~ 100$ larvae (5 dpf; $n=3$ replicates) from each group were washed five times and randomly sampled for extraction according to a previous study (Chen et al., 2013b). The samples were weighed and freeze-dried, then $5 \mathrm{ng}{ }^{13} \mathrm{C}_{12}$-labeled BDE-139 was added as recovery spike. Next, the samples were homogenized using a mixture of acetone and isooctane $(1: 1, \mathrm{v} / \mathrm{v})$, sonicated for $30 \mathrm{~min}$ and extracted three times. All extracts were passed through a $0.22 \mu \mathrm{m}$ nylon mesh filter into an autosampler vial and dried under nitrogen, the volume was added up to $500 \mu \mathrm{l}$ with isooctane, and $4 \mathrm{ng}{ }^{13} \mathrm{C}_{12}$-labeled PCB-208 was added as internal standard. Quantification of PBDEs was performed using a gas chromatograph (Agilent 6890; Agilent Technologies, Santa Clara, CA, USA) equipped with a mass-selective detector (Agilent 5975C). To check for interference or contamination from the solvent or glassware, procedural blanks were analyzed simultaneously in every batch of five samples. The recovery of ${ }^{13} \mathrm{C}_{12}$-labeled BDE-139 ranged from $87 \%$ to $98 \%$ and corrected for the results. The limit of detection was defined as a signal/noise ratio of 3 , and on average ranged from 0.01 to $0.05 \mathrm{ng}$ for the PBDEs and $0.06 \mathrm{ng}$ for tetra-BDE. Samples with concentrations below the detection limits were recorded as ND.

\section{Locomotor activity measurement}

Larval swimming behavior was monitored using a Video-Track system (ViewPoint Life Sciences, Montreal, Canada) following previously described methods (He et al., 2011). Larvae (5 dpf) were placed into 24-well microplates (one larva per well) in water, and were allowed to acclimatize for 10 min before their swimming speed was monitored. The temperature was kept at $28^{\circ} \mathrm{C}$. Larval swimming behavior was monitored under continuous light (30 $\mathrm{min})$ and light/dark transition stimulation $(5 \mathrm{~min}$ light $/ 5 \mathrm{~min}$ dark $/ 5$ min light $/ 5$ min dark) to examine their swimming speed and reactions to changes of light. A total of 32 larvae of each treatment were measured, and the data (frequency of movements, distance travel and total duration of movements) were collected every $30 \mathrm{~s}$ and further analyzed using Open Office 2.4 software (http://www.openoffice.org).

\section{Whole-mount immunofluorescence}

The immunofluorescence was carried out based on a previous method (Elsalini \& Rohr, 2003). Twenty larvae from each group ( $n=4$ replicates) were collected and fixed in 4\% paraformaldehyde overnight at $4{ }^{\circ} \mathrm{C}$, then rinsed thoroughly in phosphate-buffered solution (PBS; $137 \mathrm{~mm} \mathrm{NaCl}, 2.7 \mathrm{~mm} \mathrm{KCl}, 10 \mathrm{~mm} \mathrm{Na}{ }_{2} \mathrm{HPO}_{4}$, $2 \mathrm{~mm} \mathrm{KH}_{2} \mathrm{PO}_{4}, \mathrm{pH}$ 7.4) and dehydrated in a graded methanol series $(25,50,75$ and $100 \%)$, stored in methanol at $-20^{\circ} \mathrm{C}$. Larvae were incubated in $3 \mathrm{ml} \mathrm{10 \%} \mathrm{H}_{2} \mathrm{O}_{2}$ in methanol overnight at room temperature for bleaching and to block endogenous peroxidases, then $10 \mathrm{ml}$ PBS-T (PBS containing 0.3\% Tween-20) were added, mixed and incubated for a further $24 \mathrm{~h}$ at room temperature. The larvae were washed five times with PBS-T thoroughly and blocked for $2 \mathrm{~h}$ with $2 \%$ bovine serum albumin (BSA) in PBS-T at $37^{\circ} \mathrm{C}$, then incubated in $2 \%$ BSA in PBS-T with primary antibody against HT (1 : 500) for one night at $4{ }^{\circ} \mathrm{C}$. The rabbit polyclonal anti-5HT (ImmunoStar, Hudson, WI, USA) have been verified to be reactive with zebrafish in immunofluorescence (Airhart et al., 2012). After a thorough wash with PBS-T, the larvae were incubated in $2 \%$ 
BSA in PBS-T containing Cy3-labeled goat antirabbit (Beyotime, Jiangsu, China) $(1: 500)$ for $2 \mathrm{~h}$ at $37^{\circ} \mathrm{C}$. After washing five times for $30 \mathrm{~min}$ each with PBS-T and then washed three times for 30 min each with PBS, the larvae were imaged using a two photon laser scanning system (NOL-LSM 710; Zeiss, Jena, Germany).

\section{Serotonin (5-hydroxytryptamine) analysis}

5-HT measurement was performed as described previously (Sallinen et al., 2009). Fifty larvae from each group ( $n=4$ replicates) were collected and sonicated in $200 \mu \mathrm{l}$ of $0.1 \mathrm{M}$ perchloric acid. After centrifugation for $10 \mathrm{~min}$ at $10000 \mathrm{~g}$ at $4{ }^{\circ} \mathrm{C}$, the supernatants were filtered with a $0.22 \mu \mathrm{m}$ polyvinylidene difluoride membrane. 5-HT content was determined using high-performance liquid chromatography equipped with an ESA Colochem 5600A electrochemical detector (ECD; ESA, Chelmsford, MA, USA). Reversed phase chromatography was adopted with a $C_{18}$ column $(250 \times 3 \mathrm{~mm}$, $5 \mu \mathrm{m})$. The mobile phase consisted of methyl alcohol, acetonitrile and phosphate buffer solution (ratio $4: 7: 89$ ), and was maintained at a flow rate of $0.8 \mathrm{ml} \mathrm{min}{ }^{-1}$. The sample concentrations were obtained by referring to calibration curves of 5-HT standards, and expressed as $\mathrm{ng} \mathrm{g}^{-1}$ wet weight (ww).

\section{Protein extraction and Western blot analysis}

Protein extraction and Western blot analysis were performed as previously described (Chen et al., 2012a). Briefly, protein from $\sim 200$ zebrafish larvae from each group ( $n=4$ replicates) was extracted using commercial kits (Key-GEN BioTECH, Nanjing, China) according to the manufacturer's instructions, and the concentration was measured by the bicinchoninic acid method. Fifty micrograms of each protein sample was loaded on to a $6 \%$ or $12 \%$ sodium dodecyl sulfate-polyacrylamide gel electrophoresis gel, and then electrophoretically transferred to polyvinylidene difluoride membranes (Sigma-Aldrich). The membrane was blocked with 5\% BSA in Tris-buffered saline (10 mm Tris, $150 \mathrm{~mm} \mathrm{NaCl}, \mathrm{pH} 8.0$ ) for $1 \mathrm{~h}$ at $37^{\circ} \mathrm{C}$ and then incubated with primary antibody against the MBP (1:800; AnaSpec, Fremont, CA, USA), synapsin Ila (SYN2a; 1 : 1000; Synaptic Systems, Göttingen, Germany) or glyceraldehyde 3-phosphate dehydrogenase $(1: 1000)$ at $4{ }^{\circ} \mathrm{C}$ overnight. All primary antibodies were suitable for use in zebrafish (Chen et al., 2012b). The blots were washed five times for $10 \mathrm{~min}$ each with Tris-buffered saline Tween-20, incubated with goat antirabbit ( $1: 5000)$ horseradish peroxidase-conjugated affinipure secondary antibodies
(Proteintech, Wuhan, China) at $37^{\circ} \mathrm{C}$ for $1 \mathrm{~h}$ and then visualized with enhanced chemiluminescence using a quantitative Western blot imaging system (FluorChem Q; Alpha Innotech, San Leandro, CA, USA).

\section{RNA isolation and quantitative real-time polymerase chain reaction}

Whole-body of zebrafish larvae were homogenized (30 larvae for each replicate, $n=4$ replicates), and total RNA was extracted using Trizol Reagents (Takara) according to the manufacturer's instructions. The quality and purity were examined by $1 \%$ agarose-formaldehyde gel electrophoresis with ethidium bromide staining and $260 / 280 \mathrm{~nm}$ ratios, and concentrations were determined using a NanoDrop 2000 spectrophotometer (Thermo Fisher Scientific, Wilmington, DE, USA). First-strand cDNA was synthesized using a PrimeScript RT reagent kit (Takara) according to the manufacturer's instructions. The transcriptional levels of genes involved in neural development (shha, fgf8, wnt 1) and genes encoding the rate-limiting enzyme for serotonin synthesis, tryptophan hydroxylase (tph1, tph2, tphr), for serotonin transporters (serta and sertb) as well as for 5-HT1A receptors (htr1aa, htr $1 a b)$. The primer sequences were obtained using the online Primer 3 program (http://primer3.ut.ee/), and are listed in Table 1. The ribosomal protein L8 (rp/8), which did not vary upon DE-71 exposure (data not shown), was selected as the housekeeping gene. Quantitative RT-PCR was carried out using an All-in-One qPCR Mix (GeneCopoeia) and analyzed on an $\mathrm{ABI}$ 7300 System (PerkinElmer Applied Biosystems, Foster City, CA, USA). The change in mRNA expression was analyzed using the $2^{-\Delta \Delta \mathrm{Ct}}$ method.

\section{Molecular docking}

Molecular docking studies were performed using the LibDock module, and binding energies were calculated for all docked compounds using Discovery Studio 4.0 (Accelrys Software, San Diego, CA, USA) with standard protocols. The crystal structure of FGF8 (2FDB), SHH (2ROT) and HTR1B (4IAQ) were obtained from the RCSB protein data bank (http://www.pdb.org), and used for docking modeling after preparation. For ligand preparation, the three-dimensional structures of the PBDE congeners were obtained from the PubChem Substance database (http://www.ncbi. nlm.nih.gov/pcsubstance) and optimized with the MMFF94 method.

\begin{tabular}{|c|c|c|c|}
\hline Gene name & Sense primer $\left(5^{\prime}-3^{\prime}\right)$ & Antisense primer $\left(5^{\prime}-3^{\prime}\right)$ & GenBank accession no. \\
\hline rpl8 & TTGTTGGTGTTGTTGCTGGT & GGATGCTCAACAGGGTTCAT & NM_200713 \\
\hline shha & GCAAGATAACGCGCAATTCGGAGA & TGCATCTCTGTGTCATGAGCCTGT & NC_007118 \\
\hline fgf8 & CTATGCTCAGGTAACCATTCA & TATAAGCTTGGCATGAACGTC & NC_007124 \\
\hline wnt1 & ATGTAGGCTGATGCTTG & GTCGGCTCGGAAACTGCA & NC_007134 \\
\hline tph1 & TCTGTGAACTCTACGTGTGG & CACTGGGAGCATCAGACG & AF548566 \\
\hline tph2 & ATCCATCCTTGСTCTCCAAC & TCTGTGAACTCTACGTGTGG & NM_214795 \\
\hline tphr & AGATCCCATACCACACGTAGAG & CGGTTCAGGAGTGTAAAGAGG & $A B 125219$ \\
\hline serta & ACCACCAGAGTCCTAAATGTTCCAG & СTCTTCCTTCATCTGTGTGCCTTCC & NM_001039972 \\
\hline sertb & AACCCTAACAGCAGTCCTCA & GGCCTCACCGTCACACAATA & NM_001177459 \\
\hline htriaa & ATGAGGATGAGCGGGATGTAG & CAATCAGCCAGGACCACG & NM_001123321 \\
\hline htrlab & CTGTGTCGCCTGCACTITC & TGATCTCCAAAGACTCGCCG & NM_001145766 \\
\hline
\end{tabular}




\section{Statistical analysis}

All data are expressed as mean \pm SEM. The normality and homogeneity of variance of date were verified using Kolmogorov - Smirnov and Levene's tests. Differences between the control and exposure groups were evaluated by one-way analysis of variance followed by Tukey's test using SPSS 13.0 software (SPSS, Chicago, IL, USA). $P<0.05$ was considered statistically significant.

\section{Results}

\section{Developmental toxicity associated with DE-71 exposure}

Exposure to DE-71 did not affect embryo hatching rate, survival and growth parameters (body weight and length; data not shown). The overall hatching rates were $>90 \%$, and survival rates were $>95 \%$. A non-significant trend for an increase in the malformation rate was found in the exposure groups compared with the control group (data not shown).

\section{Concentration of DE-71 in exposed zebrafish larvae}

The congeners, including BDE-28, 47, 71, 99, 153 and 154, were detected in DE-71-exposed zebrafish larvae, with BDE-47 being the predominant one, followed by BDE-99. The detected total contents of PBDEs were $27.26 \pm 1.03,94.67 \pm 2.03,331.70 \pm 12.15$ and

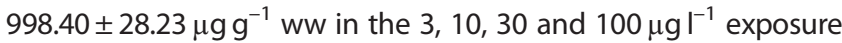
groups, respectively (Table 2). No PBDEs were detected in the control group.

\section{Locomotor activity is decreased in DE-71-exposed larvae}

The locomotion-traced zebrafish larvae (5 dpf) were monitored during continuous light and during a light-dark transition stimulation period. Under continuous light condition, the speed was significantly decreased by $15.3 \%$ and $20.0 \%(P<0.001)$ in the 30 and $100 \mu \mathrm{gl}^{-1}$ exposure groups, respectively, compared with the control (Fig. 1A).

Figure $1(B)$ and $(C)$ show the distance moved and the swimming speed of the larvae during the light-dark stimulation experiment, respectively. The total distance moved (Fig. 1B) and the average swimming speed of the larvae (Fig. 1C) during both the light and dark period were significantly decreased after exposure to 30 and $100 \mu \mathrm{gl}^{-1} \mathrm{DE}-71 \quad(P<0.001)$ compared with those of the control.
Exposure to DE-71 reduces larval 5-hydroxytryptamine immunoreactivity neuron and 5-hydroxytryptamine contents

Whole-mount immunofluorescence staining was performed using 5-HT antibody. The results demonstrated that 5-HT immunoreactivity neurons were distributed in the optic tecum and rhombencephalon, and strongly located laterally in the hindbrain (arrows) from zebrafish larvae in control group (Fig. 2A). In contrast, 5-HT immunoreactivity neurons declined in the optic tecum and rhombencephalon regions in zebrafish larvae exposed to $100 \mu \mathrm{gl}^{-1}$ DE-71 for 5 days (Fig. 2B).

Moreover, the whole-body content of $5-\mathrm{HT}$ was significantly reduced in a concentration-dependent manner and showed significant differences in the larvae exposed to 10,30 and $100 \mu \mathrm{gl}^{-1} \mathrm{DE}$ 71 by $29.8 \%, 34.1 \%$ and $46.6 \%$, respectively, compared with the solvent control (Fig. 3).

\section{Exposure to DE-71 reduces protein expression levels of myelin basic protein and synapsin Ila}

The protein expression levels of MBP and SYN2a in DE-71-exposed zebrafish were examined by Western blot. There was a concentration-dependent reduction of MBP and SYN2a (Fig. 4) in exposed embryos. Specifically, MBP was significantly reduced by $43.6 \%$ and by $52.3 \%$ in the 30 and $100 \mu \mathrm{gl}^{-1}$ exposure groups (Fig. 4A,B), respectively, whereas SYN2a was significantly reduced by $29.8 \%$ and by $37.9 \%$ in the 30 and $100 \mu \mathrm{gl}^{-1}$ exposure groups (Fig. 4C,D), respectively, compared with those of the control group.

\section{Exposure to DE-71 is associated with decreased mRNA ex- pression of genes involved in serotonergic signaling}

The measured gene transcriptional profiles are shown in Fig. 5. The transcripts of shha, fgf8 and wht1 were significantly downregulated 1.6-, 1.7- and 1.9-fold; 1.8-, 1.6- and 2.1-fold; 1.9-, 2.1- and 2.1 -fold in the 10,30 and $100 \mu \mathrm{gl}^{-1}$ groups, respectively. The mRNA level of tph1 was significantly downregulated 1.5-, 1.4and 1.5 -fold in the 10,30 and $100 \mu \mathrm{gl}^{-1}$ groups, respectively. The tph2 gene transcription was significantly downregulated 1.2-, 1.2, 2.1- and 2.1-fold in the 3,10, 30 and $100 \mu \mathrm{gl}^{-1}$ groups, respectively; the gene transcriptions of tphr, serta, sertb and htr $1 a b$ were significantly downregulated 1.4- and 1.8-fold, 1.7- and 1.7-fold, 1.8- and 2.3-fold, 2.0- and 2.1-fold in the 30 and $100 \mu \mathrm{gl}^{-1}$ groups respectively. The htr1aa gene transcription was significantly downregulated 1.7 -fold in the $100 \mu \mathrm{gl}^{-1}$ group.

Table 2. PBDE congeners measured in zebrafish larvae after exposure to different concentrations of $\mathrm{DE}-71\left(0,3,10,30 \mathrm{or}^{100} \mu \mathrm{gl} \mathrm{I}^{-1}\right)$ for 5 days

\begin{tabular}{lcccrr} 
PBDE concentration $\left(\mu \mathrm{g}^{-1}\right)$ & 0 & \multicolumn{1}{c}{3} & 10 & 30 & 100 \\
\hline BDE-28 & ND & $0.02 \pm 0.00$ & $0.04 \pm 0.00$ & $0.11 \pm 0.01$ & $3.19 \pm 0.42$ \\
BDE-47 & ND & $26.46 \pm 0.95$ & $87.54 \pm 1.66$ & $288.50 \pm 11.21$ & $901.20 \pm 24.75$ \\
BDE-71 & ND & $0.05 \pm 0.00$ & $0.06 \pm 0.00$ & $0.11 \pm 0.00$ & $0.58 \pm 0.04$ \\
BDE-99 & ND & $0.38 \pm 0.08$ & $6.36 \pm 0.50$ & $41.79 \pm 0.96$ & $91.95 \pm 3.18$ \\
BDE-153 & ND & $0.05 \pm 0.00$ & $0.17 \pm 0.01$ & $0.28 \pm 0.00$ & $0.41 \pm 0.01$ \\
BDE-154 & ND & $0.31 \pm 0.00$ & $0.51 \pm 0.01$ & $0.90 \pm 0.02$ & $1.06 \pm 0.00$ \\
SPBDE & ND & $27.26 \pm 1.03$ & $94.67 \pm 2.03$ & $331.70 \pm 12.15$ & $998.40 \pm 28.23$
\end{tabular}

$\mathrm{ND}$, below the detection limits; PBDE, polybrominated diphenyl ether.

Values $\left(\mathrm{ng} \mathrm{g}^{-1} \mathrm{ww}\right.$ ) are expressed as mean \pm SEM of three replicate samples. 


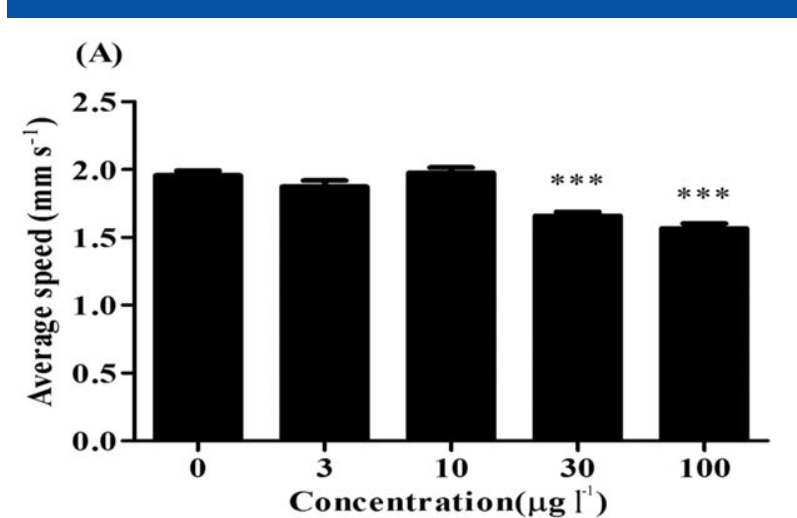

(B)

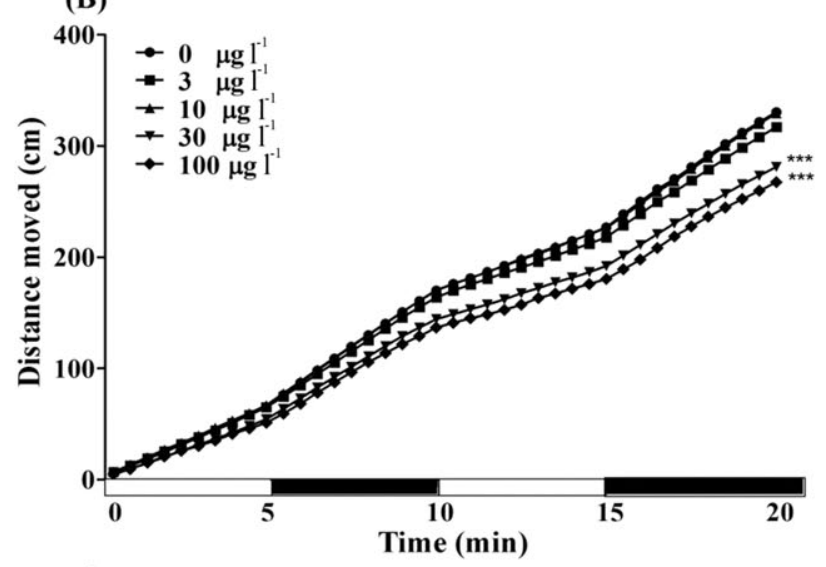

(C)

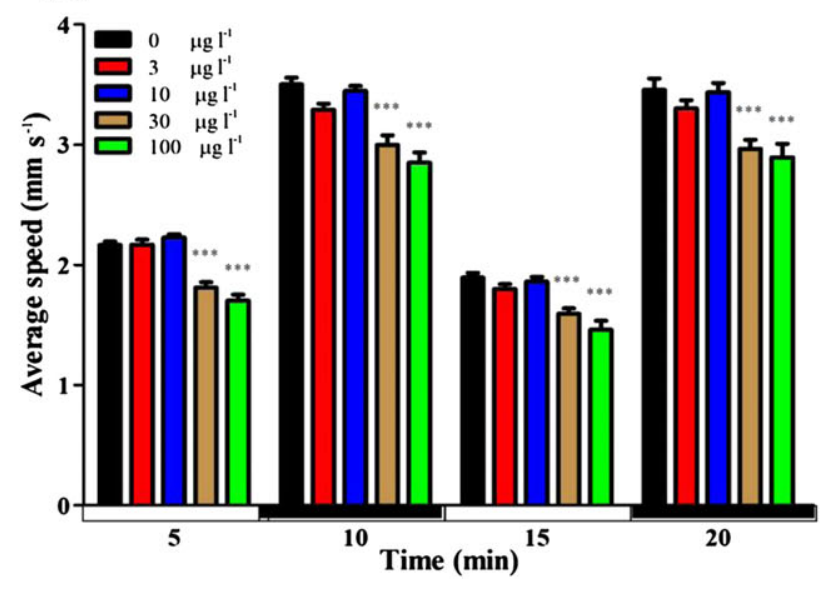

Figure 1. Abnormal locomotor behavior in zebrafish larvae exposed to DE-71. Locomotor behavior was assessed in zebrafish larvae exposed to 0 , $3,10,30$ or $100 \mu \mathrm{gl}^{-1} \mathrm{DE}-71$ for 5 days. The following parameters were measured: average swimming speed during a continuous light test (A); distance moved (B) and average swimming speed (C) of the larvae during a lightdark-light-dark photoperiod stimulation test. Data are expressed as mean \pm SEM of four replicates (eight larvae per replicate) in $30 \mathrm{~s}$ intervals; ${ }^{* * *} P<0.001$ indicate significant difference between the exposed larvae and the control group.

\section{Interactions between polybrominated diphenyl ethers and serotonergic related proteins indicated by molecular docking}

No poses were generated for the PBDE congeners with the SHH protein; hence, no data for their binding energies were obtained. The binding energy of the docked PBDE congeners with FGF8 and HTR1B are shown in Table 3. All congeners detected in the present study could bind to FGF8, with binding energies ranging from -88.81 to $-69.79 \mathrm{kcal} \mathrm{mol}^{-1}$. The congeners of BDE-47, BDE-28, BDE-99 and BDE-154 were found to bind to HTR1B, with binding energies ranging from 6.72 to $25.42 \mathrm{kcal} \mathrm{mol}^{-1}$.

The binding modes of the docked PBDE congeners were further analyzed and are shown in Fig. 6 . The protein-ligand interactions depict several residues (Arg59, Arg3255 and Asn2173) and water molecules ( $\mathrm{HOH} 114$ and $\mathrm{HOH} 125)$ forming $\mathrm{H}$-bonds with bromine atoms of BDE154, and residues (Arg3255 and Asn2173) forming the $\mathrm{H}$-bond with the oxygen atom (Fig.6A). Analyses of the FGF8BDE154 complex also showed $\pi-\pi$ stacking interactions mediated by Phe3352 and $\pi$-cation interactions by Arg 59, Arg170 and Arg 3255. No H-bonds or $\pi$ interaction pairs were found, and van der Waals force plays a major role within the HTR1B-BDE47 complex (Fig. 6B).

\section{Discussion}

The aim of this study was to validate the possible effects of PBDEs on the neural developmental and serotonergic systems by an in vivo zebrafish model, and to predict the molecular target through in silico tools. Our results demonstrated that embryonic exposure to DE-71 damaged early development and caused impairment of the genes and proteins involved in serotonergic signaling in zebrafish larvae, and the docking results suggested FGF8 as a possible target of PBDEs congeners.

First, we determined the behavioral changes as an endpoint of neurotoxicity upon DE-71 exposure, and observed hypoactivity, as indicated by reduced trace and swimming speed in continuous light and light-dark transition stimulation in zebrafish larvae exposed to 30 and $100 \mu \mathrm{gl}^{-1} \mathrm{DE}-71$. This observation is in agreement with the altered behavioral activity previously reported in PBDE-exposed zebrafish larvae (Chen et al., 2012b; He et al., 2011). In addition, the detected congeners, BDE-47, BDE-99 and BDE-153 have also been shown to be related to alterations of spontaneous behavior (locomotion, rearing and total activity). Importantly, although the waterborne concentrations of DE-71 used in this study were higher than those measured in the water, the body burden of total PDBEs in exposed zebrafish larvae in the $100 \mu \mathrm{gl}^{-1}$ group (998.40 $\mathrm{ng} \mathrm{g}^{-1} \mathrm{WW}$ ) was comparable with those in fish collected from PBDE-contaminated water $\left(1088 \mathrm{ng} \mathrm{g}^{-1} \mathrm{ww}\right.$ ) (Luo et al., 2007). Thus, our results confirmed the neurotoxicity upon DE71 exposure and suggested a potential risk for wild fish in PBDE-contaminated water.

To analyze further the underlying mechanism, we investigated the variation of important genes and proteins regarding early neural development. The transcriptional levels of fgf8, shha and wnt1, showed similar, decreasing trends in zebrafish larvae exposed to DE-71 concentrations $\geq 10 \mu \mathrm{gl}^{-1}$. These observations suggest that PBDE exposure impairs the early development procedure such as induction of the neural plate, proliferation and differentiation of neuronal progenitors. Moreover, our Western blot analysis revealed significantly reduced contents of MBP and SYN2a, both of which are involved in neuronal morphogenesis. Previous studies have reported transcriptional changes of both $m b p$ and syn $2 a$ upon PBDE exposure in zebrafish (Chen et al., 2012b), providing further support for our results. These results provided evidence for the neural development toxicity by DE-71. On this basis, we employed a docking simulation to predict the possible target among the affected modulators by evaluating their binding affinities and 


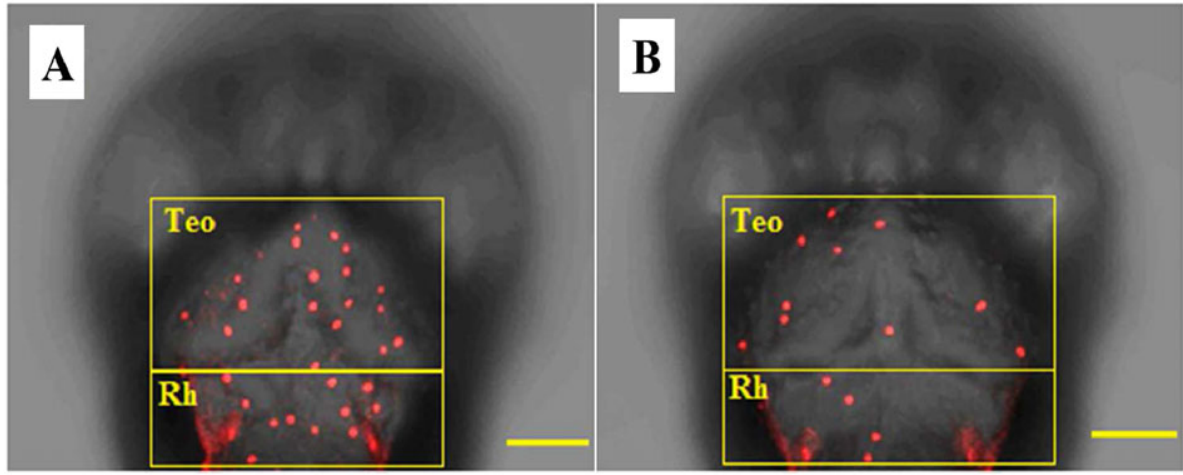

Figure 2. DE-71 exposure is associated with declined 5-HT-ir cells. 5-HT-ir neurons are observed distributed in the Teo and Rh from zebrafish larvae in the control group (A). In contrast, 5-HT-ir neurons declined in Teo and Rh regions in zebrafish larvae exposed to $100 \mu \mathrm{g} \mathrm{I} \mathrm{I}^{-1} \mathrm{DE}-71$ for 5 days (B). Images were taken dorso to the ventral. Scale bar is $100 \mu \mathrm{m}$. 5-HT-ir, 5-hydroxytryptamine (serotonin) immunoreactivity; Rh, rhombencephalon; Teo, optic tecum.

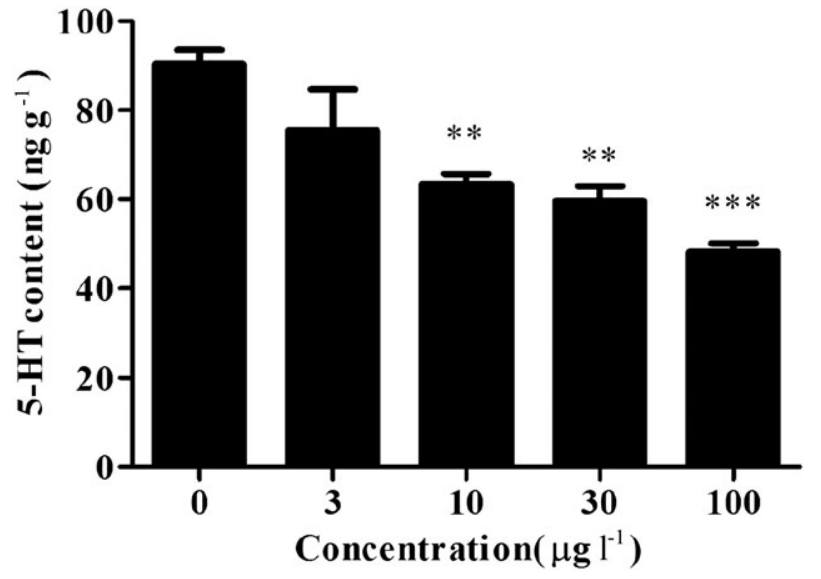

Figure 3. DE-71 exposure is associated with a reduced whole-body content of serotonin. The concentration of 5-HT was measured in zebrafish larvae exposed to $0,3,10,30$ and $100 \mu \mathrm{gl} \mathrm{I}^{-1}$ DE-71 for 5 days. $n=4$ replicate samples; each sample contained 50 larvae; data are expressed as mean $\pm \mathrm{SEM} ;{ }^{* *} P<0.01$ and ${ }^{* * *} P<0.001$ indicate significant differences between the exposed larvae and the control group. 5-HT-ir, 5-hydroxytryptamine (serotonin).

interacting modes with PBDE congeners. Owing to the availability of crystal structures, FGF8 and SHH were chosen and tested. The PBDE congeners showed poor affinities with $\mathrm{SHH}$, as no docking poses were generated by docking simulation. In contrast, all congeners detected in our study could bind significantly to FGF8, as indicated by their negative binding energies. This could also be supported by observations of the $\mathrm{H}$-bonds and $\pi$ interaction pairs within the protein-ligand complex. Therefore, we assume that FGF8 could be the potential molecular target of PBDEs congeners. Despite the limited cognition of the complex mechanism(s) among the modulators, a functional link between FGF8 and WNT1 signaling through an autoregulatory loop (Chilov et al., 2010) and an regulatory relationship between FGF8 and MBP (Chen et al., 2013a) have been proved. Therefore, it was possible that the observed alterations of these genes and proteins were attributed to the alterations of fgf8 signaling.

Considering the regulatory role of $f g f 8$, shh and wht 1 signaling in serotonergic neurogenesis (Teraoka et al., 2004), we performed whole-mount immunofluorescence assay using 5-HT antibody, and the results demonstrated a decline in the number of serotonin-positive neurons in the brain of zebrafish larvae. In accordance with this, 5-HT levels determined by highperformance liquid chromatography showed a dosedependent reduction upon PBDE exposure concentrations in zebrafish larvae. These results may indicate impairment of serotonergic neurogenesis, which resulted in decreased 5-HT levels in zebrafish larvae upon DE-71 exposure. Although the specific role of the serotonergic system in the control of behavior is not clear, some correlations between the locomotion and 5-HT levels have been established. For example, 5-HT-depleted mice exhibited hypoactivity in spontaneous locomotor activity and a less anxious performance in light-dark box exploration, and in zebrafish, serotonin depletion resulted in significantly less movement (Airhart et al., 2012; Schaefer et al., 2009), which is similar to the behavioral observations in our study. Thus, our results indicate that the behavioral changes of zebrafish larvae upon PBDE exposure were related, at least partially, to the decreased 5-HT levels.

In addition, the transcripts of genes involved in the synthesis and neurotransmission of 5-HT were determined, and the results suggested that the genes encoding tryptophan hydroxylase (tph1, tph2, tphr) were significantly suppressed. Aroclor 1254, a PCB mixture, has been demonstrated to inhibit hypothalamic tryptophan hydroxylase activity and to result in decreased hypothalamic 5-HT concentrations in the Atlantic croaker (Micropogonias unulatus) (Khan \& Thomas, 2001). Thus, inhibited transcription levels of tph1, tph2 and tphr suggested reduced synthesis of 5-HT, and this may explain the decreased 5-HT levels in zebrafish larvae exposed to PBDEs. In a previous study, embryonic exposure to pchlorophenylalanine, an inhibitor of $\mathrm{TPH}$, resulted in decreased transcripts of htrla in the brain and spinal cord, and in reduced SERT in the spinal cord of zebrafish (Airhart et al., 2012). Similarly, the gene transcripts of $h t r 1 a a / b$ and serta/b were also found to be inhibited in addition to the decreased tph transcripts in DE-71-exposed zebrafish larvae. Taken together, the transcriptional suppression of 5-HT1A receptors (htr1aa, htr1ab) and serotonin transporters (serta and sertb) suggests a dysfunction in 5-HT neurotransmission caused by PBDE exposures. The results described suggest a profound toxic effect on the genes involved in serotonergic signaling upon PBDE exposures.

However, some other studies indicated a role of $5-\mathrm{HT}$ in the specification, differentiation and phenotype maintenance of the hippocampal neurons, including the serotonergic 


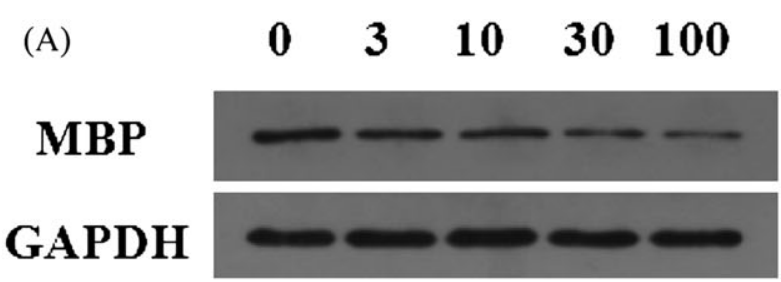

(B)

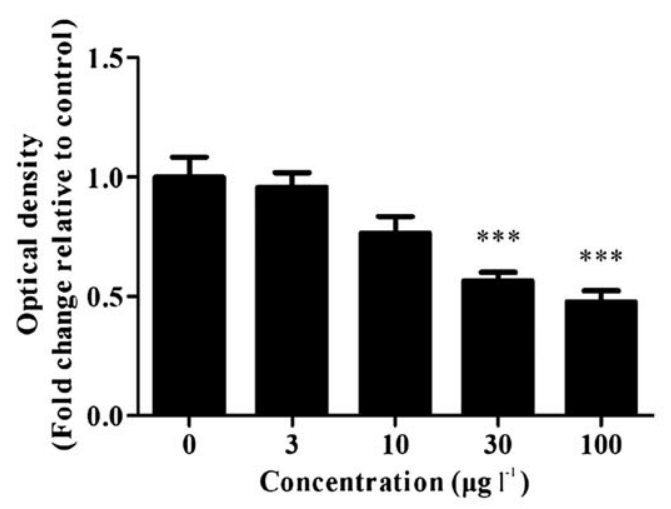

(C)

\section{$\begin{array}{lllll}0 & 3 & 10 & 30 & 100\end{array}$}

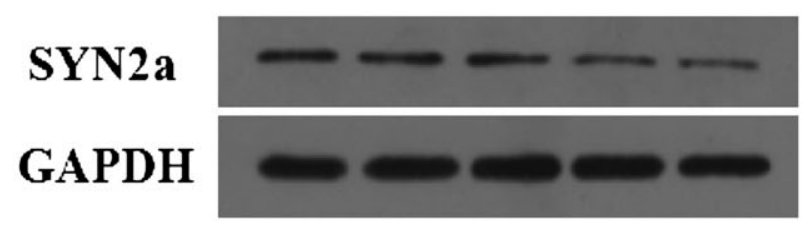

(D)

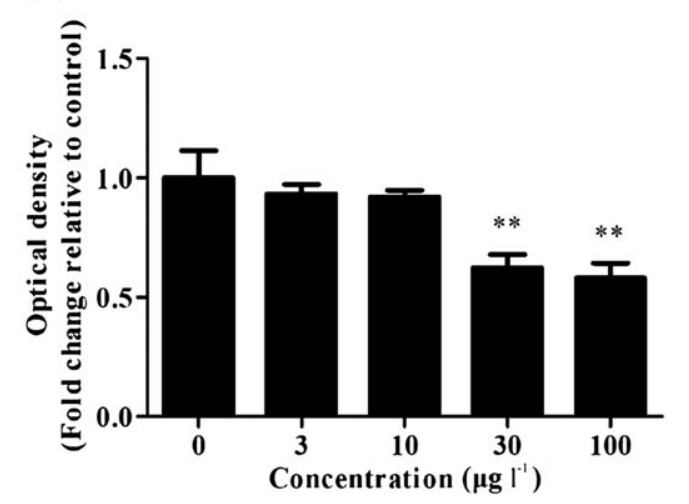

Figure 4. DE-71 exposure is associated with reduced protein expression levels of MBP and SYN2a. Western blot analysis was used to measure the protein expression level of $\operatorname{MBP}(A, B)$ and SYN2a $(C, D)$ in zebrafish larvae exposed to $0,3,10,30$ and $100 \mu \mathrm{gl}^{-1}$ of DE-71 for 5 days. Upper panel shows a representative Western blot $(A, C)$ and lower panel shows quantification of four replicate samples $(B, D)$, normalized to GAPDH expression; data are expressed as mean $\pm \mathrm{SEM}$; ${ }^{* *} P<0.01 ;{ }^{* * *} P<0.001$ indicate significant differences from the control group. GAPDH, glyceraldehyde 3-phosphate dehydrogenase; MBP, myelin basic protein; SYN2a, synapsin Ila.

system via activation of its receptors (Fricker et al., 2005), thus raised an opposite hypothesis that PBDEs could directly affect the 5-HT signaling and result in an indirect effect on the neural development. In this regard, we evaluated the binding affinities and interacting modes between PBDE

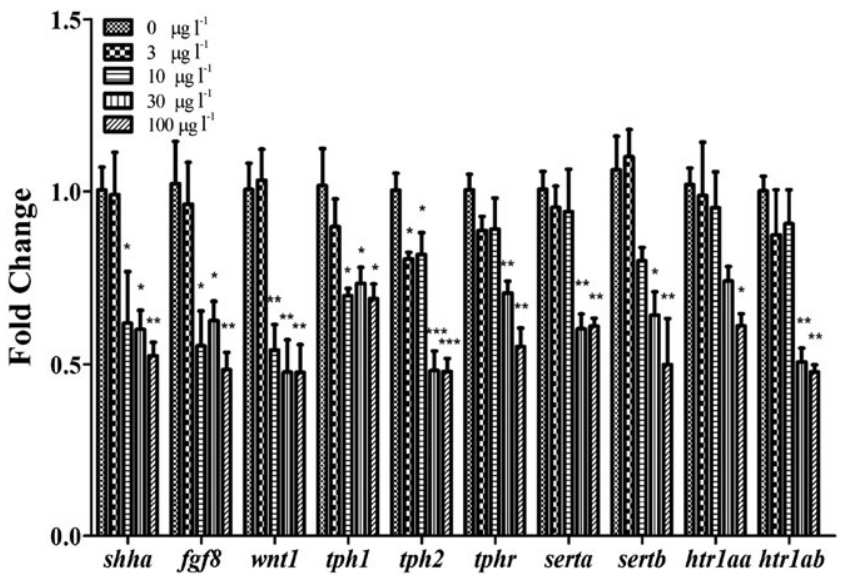

Figure 5. Altered genes expression in zebrafish larvae exposed to DE-71. The mRNA expression levels were significantly decreased following exposure to $0,3,10,30$ and $100 \mu \mathrm{gl}^{-1} \mathrm{DE}-71$ for 5 days in a concentrationdependent manner. Data are presented as mean \pm SEM $n=4$ replicate samples; each sample contained 30 larvae; ${ }^{*} P<0.05,{ }^{* *} P<0.01$, ${ }^{* *} P<0.001$ indicate significant differences between the exposed larvae and the control group.

Table 3. Binding energies of PBDE congeners as docked ligands

\begin{tabular}{|lcc|}
\hline Protein & PBDE congeners & Binding energy $\left(\mathrm{kcal} \mathrm{mol}^{-1}\right)$ \\
\hline FGF8 & BDE-47 & -77.02 \\
& BDE-71 & -88.81 \\
& BDE-28 & -81.80 \\
& BDE-99 & -83.63 \\
& BDE-154 & -76.21 \\
HTR1B & BDE-153 & -69.79 \\
& BDE-47 & 25.42 \\
& BDE-28 & 13.72 \\
& BDE-99 & 21.80 \\
PBDE, polybrominated diphenyl ether. & \\
\hline
\end{tabular}

congeners and the 5-HT receptor, HTR1B. The results indicated that PBDE congeners showed weak interactions with HTR1B, as only BDE-47, BDE-28, BDE-99 and BDE-154 could be docked into generated poses with positive binding energies. The interaction diagram also showed no $\mathrm{H}$-bond within the protein-ligand complex. Therefore, we suggest that PBDE congeners would probably not interact with this $5-\mathrm{HT}$ receptor. In addition, these results confirmed that the impaired serotonergic signaling could be secondary effects following impaired neural development.

In conclusion, the present results demonstrate that DE-71 exposure during zebrafish embryogenesis results in the repression of certain genes and proteins involved in neural development, and resulted in secondary effects including dysfunction of serotonergic synthesis and neurotransmission, and finally the reduction of 5-HT levels. The docking simulation indicated FGF8 as a possible target of PBDE congeners. Further studies employing genetic tools would be necessary to verify and understand the role of FGF8 signaling in PBDE-induced developmental neurotoxicity. 


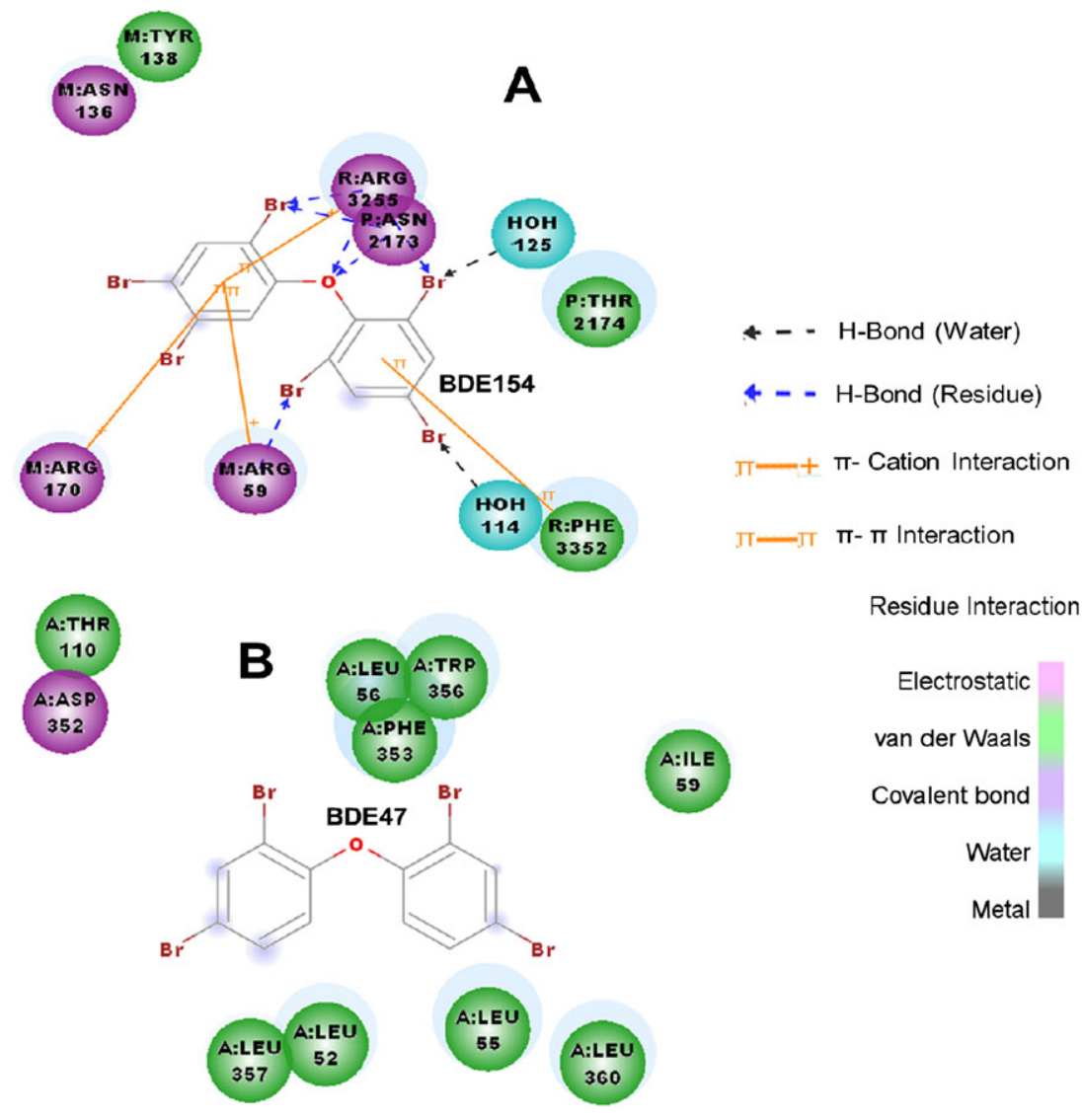

Figure 6. Interaction map depicting protein-ligand contacts in the top hits obtained by Libdock using Discovery Studio. Ligand interaction diagram showing the protein-ligand contacts for (A) FGF8-BDE154 and (B) HTR1B-BDE47, with essential amino acid residues tagged in circles.

\section{Acknowledgments}

This work was supported by the National Natural Science Foundation of China (nos. 21307153, 21237005), the National Environmental Protection Public Welfare Science and Technology Research Program of China (no. 201309047), and the Knowledge Innovation Program of the Chinese Academy of Sciences (Y25E03).

\section{Conflict of interest}

The authors did not report any conflict of interest.

\section{References}

Airhart MJ, Lee DH, Wilson TD, Miller BE, Miller MN, Skalko RG, Monaco PJ. 2012. Adverse effects of serotonin depletion in developing zebrafish. Neurotoxicol. Teratol. 34: 152-160.

Bengel D, Murphy DL, Andrews AM, Wichems CH, Feltner D, Heils A, Mossner R, Westphal H, Lesch KP. 1998. Altered brain serotonin homeostasis and locomotor insensitivity to 3,4-methylenedioxymethamphetamine ("ecstasy") in serotonin transporter-deficient mice. Mol. Pharmacol. 53 649-655.

Brosamle C, Halpern ME. 2002. Characterization of myelination in the developing zebrafish. Glia 39: 47-57.

Chen Q, Yu L, Yang L, Zhou B. 2012a. Bioconcentration and metabolism of decabromodiphenyl ether (BDE-209) result in thyroid endocrine disruption in zebrafish larvae. Aquat. Toxicol. 110: 141-148.

Chen L, Yu K, Huang C, Yu L, Zhu B, Lam PK, Lam JC, Zhou B. 2012b. Prenatal transfer of polybrominated diphenyl ethers (PBDEs) results in developmental neurotoxicity in zebrafish larvae. Environ. Sci. Technol. 46: 9727-9734.

Chen H, Chew L, Packer RJ, Gallo V. 2013a. Modulation of the Wnt/beta-catenin pathway in human oligodendroglioma cells by
Sox17 regulates proliferation and differentiation. Cancer Lett. 335: $361-371$.

Chen L, Huang Y, Huang C, Hu B, Hu C, Zhou B. 2013b. Acute exposure to $\mathrm{DE}-71$ causes alterations in visual behavior in zebrafish larvae. Environ. Toxicol. Chem. 32: 1370-1375.

Chilov D, Sinjushina N, Saarimaki-Vire J, Taketo MM, Partanen J. 2010. betaCatenin regulates intercellular signalling networks and cell-type specific transcription in the developing mouse midbrain-rhombomere 1 region. PLoS One 5: e10881.

Costa LG, Giordano G. 2007. Developmental neurotoxicity of polybrominated diphenyl ether (PBDE) flame retardants. Neurotoxicology 28: 1047-1067.

Dingemans MM, van den Berg M, Westerink RH. 2011. Neurotoxicity of brominated flame retardants: (in)direct effects of parent and hydroxylated polybrominated diphenyl ethers on the (developing) nervous system. Environ. Health Perspect. 119: 900-907.

Eddins D, Cerutti D, Williams P, Linney E, Levin ED. 2010. Zebrafish provide a sensitive model of persisting neurobehavioral effects of developmental chlorpyrifos exposure: Comparison with nicotine and pilocarpine effects and relationship to dopamine deficits. Neurotoxicol. Teratol. 32: 99-108.

Elsalini OA, Rohr KB. 2003. Phenylthiourea disrupts thyroid function in developing zebrafish. Dev. Genes Evol. 212: 593-598.

Eriksson P, Jakobsson E, Fredriksson A. 2001. Brominated flame retardants: A novel class of developmental neurotoxicants in our environment? Environ. Health Perspect. 109: 903-908.

Fricker AD, Rios C, Devi LA, Gomes I. 2005. Serotonin receptor activation leads to neurite outgrowth and neuronal survival. Brain Res. Mol. Brain Res. 138: 228-235.

Gershon MD, Tack J. 2007. The serotonin signaling system: from basic understanding to drug development for functional GI disorders. Gastroenterology 132: 397-414.

He J, Yang D, Wang C, Liu W, Liao J, Xu T, Bai C, Chen J, Lin K, Huang C, Dong Q. 2011. Chronic zebrafish low dose decabrominated diphenyl ether (BDE-209) exposure affected parental gonad development and locomotion in F1 offspring. Ecotoxicology 20: 1813-1822. 
Hirabayashi Y, Itoh Y, Tabata H, Nakajima K, Akiyama T, Masuyama N, Gotoh Y. 2004. The Wnt/beta-catenin pathway directs neuronal differentiation of cortical neural precursor cells. Development 131: 2791-2801.

Hynes M, Ye W, Wang K, Stone D, Murone M, Fd S, Rosenthal A. 2000. The seven-transmembrane receptor smoothened cell-autonomously induces multiple ventral cell types. Nat. Neurosci. 3: 41-46.

Kao HT, Porton B, Czernik AJ, Feng J, Yiu G, Haring M, Benfenati F, Greengard P. 1998. A third member of the synapsin gene family. Proc. Natl. Acad. Sci. U. S. A. 95: 4667-4672.

Khan IA, Thomas P. 2001. Disruption of neuroendocrine control of luteinizing hormone secretion by Aroclor 1254 involves inhibition of hypothalamic tryptophan hydroxylase activity. Biol. Reprod. 64: 955-964.

Kodavanti PR, Ward TR. 2005. Differential effects of commercial polybrominated diphenyl ether and polychlorinated biphenyl mixtures on intracellular signaling in rat brain in vitro. Toxicol. Sci. 85: 952-962.

Li F, Xie Q, Li X, Li N, Chi P, Chen J, Wang Z, Hao C. 2010. Hormone activity of hydroxylated polybrominated diphenyl ethers on human thyroid receptor-beta: In vitro and in silico investigations. Environ. Health Perspect. 118: 602-606.

Li X, Ye L, Shi W, Liu H, Liu C, Qian X, Zhu Y, Yu H. 2013. In silico study on hydroxylated polychlorinated biphenyls as androgen receptor antagonists. Ecotoxicol. Environ. Saf. 92: 258-264.

Luo Q, Cai ZW, Wong MH. 2007. Polybrominated diphenyl ethers in fish and sediment from river polluted by electronic waste. Sci. Total Environ. 383: 115-127.

Mariussen E, Fonnum F. 2003. The effect of brominated flame retardants on neurotransmitter uptake into rat brain synaptosomes and vesicles. Neurochem. Int. 43: 533-542.

Masson J, Emerit MB, Hamon M, Darmon M. 2012. Serotonergic signaling: multiple effectors and pleiotropic effects. WIREs Membr. Transp. Signal. 1: 685-713.
Muroyama Y, Kondoh H, Takada S. 2004. Wnt proteins promote neuronal differentiation in neural stem cell culture. Biochem. Biophys. Res. Commun. 313: 915-921.

Norton WH, Folchert A, Bally-Cuif L. 2008. Comparative analysis of serotonin receptor (HTR1A/HTR1B families) and transporter (slc6a4a/b) gene expression in the zebrafish brain. J. Comp. Neurol. 511: 521-542.

Olivier JDA, Van Der Hart MGC, Van Swelm RPL, Dederen PJ, Homberg JR, Cremers T, Deen PMT, Cuppen E, Cools AR, Ellenbroek BA. 2008. A study in male and female 5-HT transporter knockout rats: An animal model for anxiety and depression disorders. Neuroscience 152: 573-584.

Sallinen V, Torkko V, Sundvik M, Reenila I, Khrustalyov D, Kaslin J, Panula P. 2009. MPTP and MPP+ target specific aminergic cell populations in larval zebrafish. J. Neurochem. 108: 719-731.

Schaefer TL, Vorhees CV, Williams MT. 2009. Mouse plasmacytomaexpressed transcript 1 knock out induced $5-\mathrm{HT}$ disruption results in a lack of cognitive deficits and an anxiety phenotype complicated by hypoactivity and defensiveness. Neuroscience 164: 1431-1443.

Teraoka H, Russell C, Regan J, Chandrasekhar A, Concha ML, Yokoyama R, Higashi K, Take-uchi M, Dong W, Hiraga T, Holder N, Wilson SW. 2004. Hedgehog and fgf signaling pathways regulate the development of tphr-expressing serotonergic raphe neurons in zebrafish embryos. J. Neurobiol. 60: 275-288.

Viti J, Gulacsi A, Lillien L. 2003. Wnt regulation of progenitor maturation in the cortex depends on Shh or fibroblast growth factor 2. J. Neurosci. 23: 5919-5927.

Wang Y, Takai R, Yoshioka H, Shirabe K. 2006. Characterization and expression of serotonin transporter genes in zebrafish. Tohoku J. Exp. Med. 208: 267-274.

Ye W, Shimamura K, Rubenstein JL, Hynes MA, Rosenthal A. 1998. FGF and Shh signals control dopaminergic and serotonergic cell fate in the anterior neural plate. Cell 93: 755-766.

Yu L, Deng J, Shi X, Liu C, Yu K, Zhou B. 2010. Exposure to DE-71 alters thyroid hormone levels and gene transcription in the hypothalamicpituitary-thyroid axis of zebrafish larvae. Aquat. Toxicol. 97: 226-233. 